\title{
Enhancing Learning with Off-Task Social Dialogues
}

\author{
Jozef Tvarožek and Mária Bieliková \\ Faculty of Informatics and Information Technologies, \\ Slovak University of Technology, \\ Ilkovičova 3, 84216 Bratislava, Slovakia \\ \{jtvarozek, bielik\}@fiit.stuba.sk
}

\begin{abstract}
In Peoplia, a socially intelligent tutoring agent helps students learn by augmenting learning opportunities with social features. The tutoring agent engages in off-task conversations with the students before and after the instructional activities, motivating them to work with the system more successfully. We describe the tutor's architecture and early experiments in the domain of middle school mathematics. Students who engaged with the socially intelligent agent liked the system more, and attained higher learning gains.
\end{abstract}

Keywords: off-task dialogues, social intelligence, motivation, problem solving.

\section{Introduction}

Tradeoffs between motivating students vs. providing them with actual learning experiences are still researched [1]. Various approaches for improving student's motivation and learning have been proposed: addressing emotional and affective states of students [2], narrative-centered environments with story-based learning [3,4], and adaptive web-based systems $[5,6]$. The affective support seems hard to realize in practice and currently remains limited [7], and since narrative-centered story-based approaches completely alter the way teaching occurs as compared to traditional classrooms or even a typical ITS interface, their use in traditional domains such as mathematics and computer science is not exactly straightforward.

In our research we attempt to improve students' motivation in learning environments with a socially intelligent agent, the tutoring friend, which addresses social aspects beyond that of an individual student [8]. In this paper, we describe the architecture of a socially intelligent tutoring agent, and report on experiments evaluating the effects of introducing the tutoring agent into a learning environment.

The social context of individual students is important in learning, and friends engage in more extensive conversations and have been found to be more supportive and critical that non-friends [9]. On the other hand, expert human teachers watch both task-oriented performance and motivational indicators [10], and various socially intelligent interaction tactics for tutoring agents to accomplish motivational goals have been proposed [11]. Major research efforts also continue to explore politeness and its role in effective tutorial dialogue, motivating students and learning [12,13,14].

Research suggests that artificial tutors are able to maintain the appearance of social intelligence without full natural language understanding, that is currently deemed 
intractable. In our approach, the socially intelligent agent employs a finite-state dialogue management that is able to engage in simple getting-to-know conversations, after which student is guided to structured problem solving exercises. Students that engaged with the artificial tutoring friend produced better learning outcomes.

\section{The Peoplia System - Overview}

Peoplia is an interactive web-based environment that helps students to learn using various types of learning opportunities that are facilitated by a socially intelligent tutoring agent, the tutoring friend [8], see Figure 1. It features pseudo-tutor assessments with free-text answering. Questions for assessments and exercises are generated by a task generator, discouraging cheating and surface learning. Peoplia is an attempt to build an integrated environment for (1) the assessments as occurring in regular classrooms, and (2) home study with self exercises; while augmenting the available learning opportunities with social features [15]. It contains generic social features such as friends management, invitations, and updating your profile.

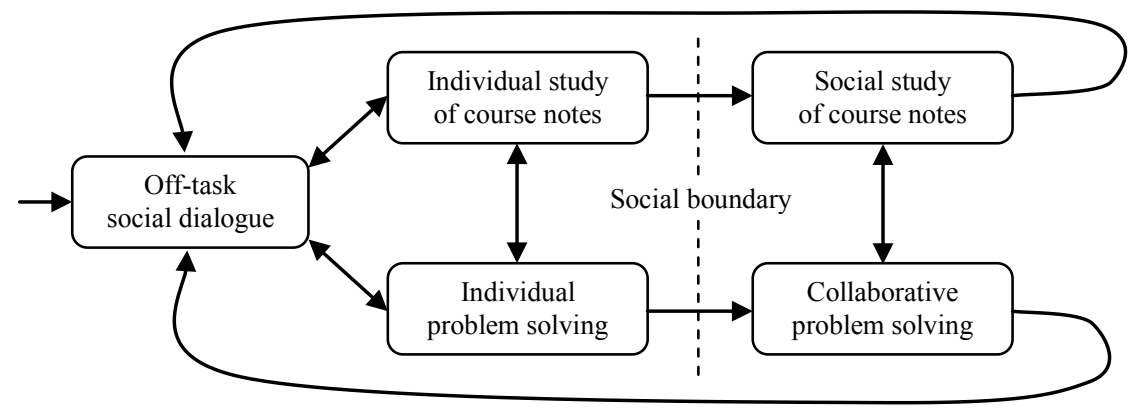

Fig. 1. Types of learning opportunities in Peoplia with admissible transitions (arrows), which are facilitated by the tutoring friend.

Learning opportunities. The system (Figure 1) features two learning types: study of course notes and problem solving, that can be engaged either individually or in collaboration, i.e. four types of learning opportunities are available in total. The most traditional form of learning support, course notes (Figure 2), is used for supporting conceptually difficult domains such as theoretical computer science, for which developing a large set of pseudo-tutor problems may not be feasible or desirable. Course materials are represented in the same rich content format as problem descriptions. Transferring existing materials into this format does not require use of complex features of the format and thus requires only a small additional effort. In social mode, course materials are augmented by facilities for highlighting important concepts, and assigning sticky notes that enable to unwind a Q\&A conversation.

For problem solving, students work on structured exercises that start with an initial question. Upon providing a wrong answer additional questions or scaffolding hints are administered to the student [16]. Difficulties of following these solution paths are statistically calibrated to allow for adaptive selection of tasks and a premature finish 
of administering a subtask that becomes too hard for the current level of student's ability.

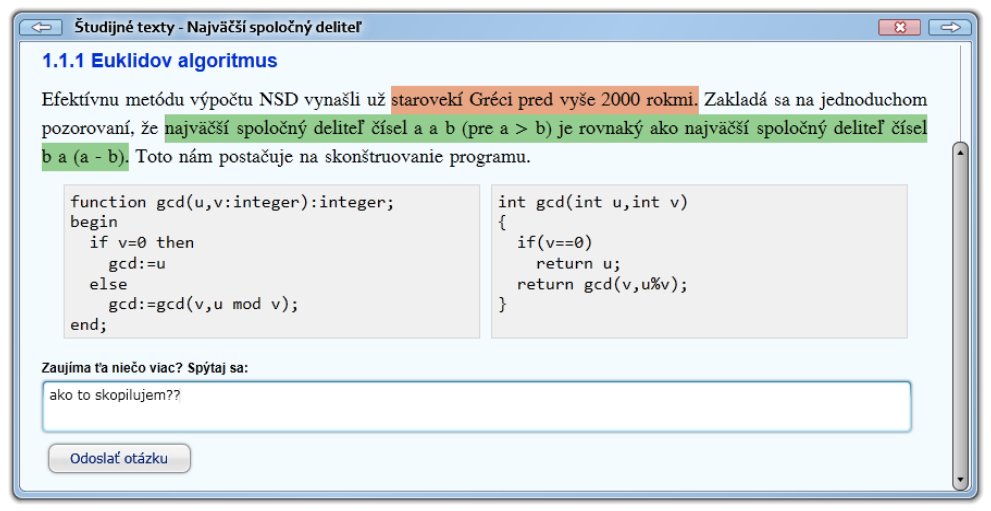

Fig. 2. Sample of course notes with highlights, sticky notes (not shown), and an option to ask for further assistance.

In collaborative mode (Figure 3), individual problem solving is enhanced by synchronous collaboration features: (1) instant messaging, (2) voting for the most agreeable answer in the team, and (3) a multi-user interface version of interactive components that are specified in the question description.

Student answers are graded (matched to the predefined set specified in the problem description) by the two-stage grading process mentioned earlier. A single human grader is capable of grading answers generated by a class of 20 students without any noticeable lag in responses to the students [16].

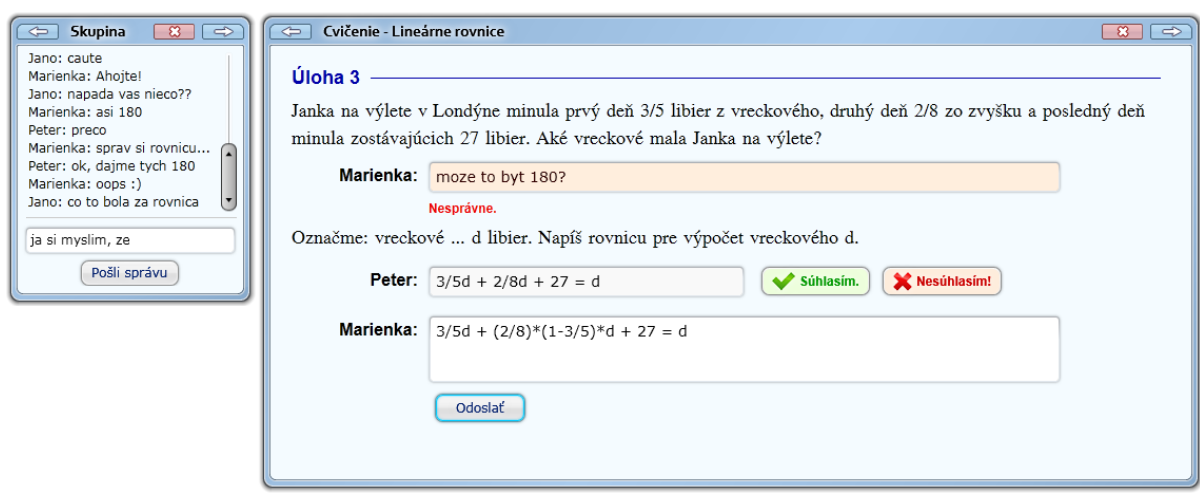

Fig. 3. Collaborative problem solving - free-text answer with voting mechanism.

Social intelligence in Peoplia. The surface features of Peoplia make it a generic shell for an interactive pseudo-tutor environment, with the accent on ways that the social dimension augments the features of students' learning experience. The tutoring friend guides students to structured learning opportunities using the off-task social dialogues. Social dialogues are designed to be task-agnostic and can be scripted separately from the domain content, making it a tractable problem. Nevertheless, the 
tutoring friend influences the transitions between different learning activities such that the reasons for the transition become clear to the student.

Transitions are governed by rules that can recommend a good course of action for the student at any given moment. For example, when an examination is imminent, the student is advised to work on exercises from a similar problem set. Rules for facilitating the transitions get more involved when the social boundary is crossed (Figure 1), as other people are a valuable resource with which the tutoring friend can "negotiate". It is not possible for a human student to cross this boundary at will, and the transition must be facilitated by the tutoring friend. For example, when a student repeatedly demonstrates incompetent behavior (in terms of social/task abilities) the tutoring friend can refuse to put him in a group that would probably only impair the work of others due to his unfit behavior. On the positive end, when a student of low ability who is otherwise completely polite does seem to have hit an impasse, he might get paired up with a student of high ability who would be (through negotiations) expected to provide a very helpful hand for at least a short amount of his time.

The appearance of social intelligence in the off-task conversations is based on a finite-state dialogue manager. For the sake of experiments a dialogue manager capable to extract student's hobbies was developed (Figure 4). Dialogues were limited in length so that its "human" traits do not become readily apparent, while the tutor's dialogue capability is improved offline from dialogue transcripts by content authors.

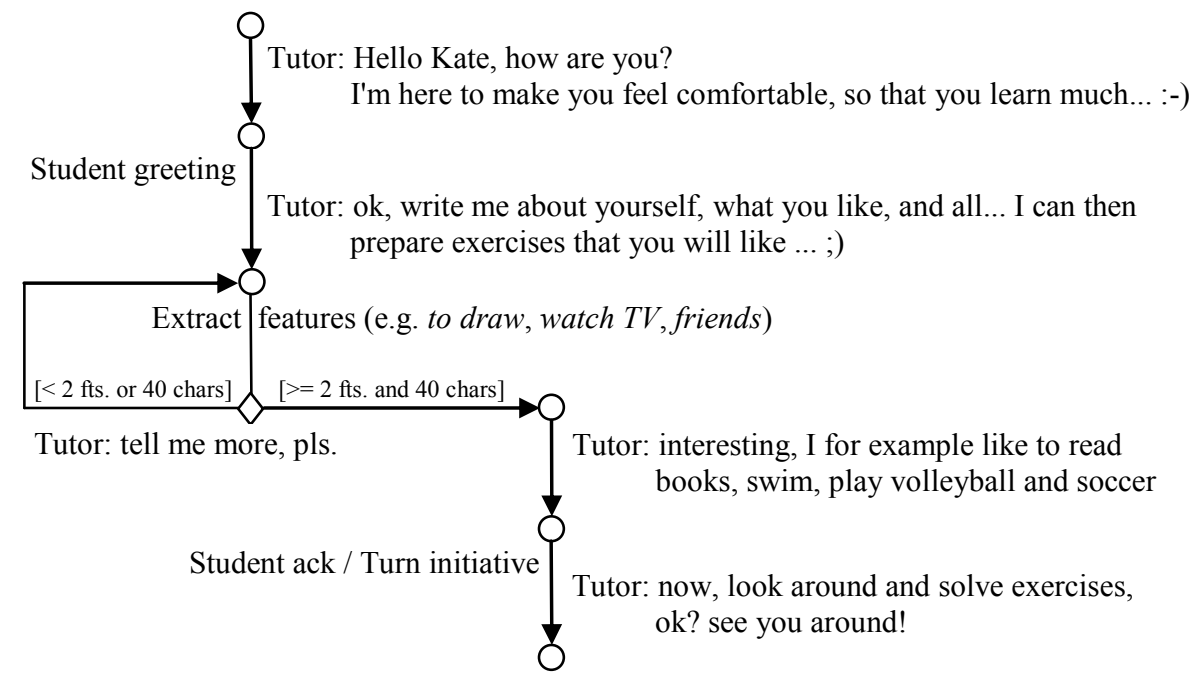

Fig. 4. Finite-state dialogue manager used for extraction of student's hobbies.

Technical considerations. On the server side, data persistence works as a large writeback cache allowing for near zero database accesses for most of the clients' requests. The student environment is a rich client implemented in the Microsoft Silverlight framework and communicates with the server over the TCP layer in duplex mode, which combined with the caching functionality enables sub-100 ms latencies for common students' actions within the environment even though most of the decisions are made on the server. 


\section{Evaluation and Discussion}

In previous experiments when middle school students were tasked to work with the system during an algebra class [8], we observed that students reveal on average 1.56 (st.dev 1.75) features about themselves in a social conversation with the tutoring friend, and only about $56 \%$ of the students engage with the tutoring agent at all.

In this paper, we analyze post hoc the learning outcomes of students that engaged vs. those that did not engage in social conversation with the tutoring friend. Total of 16 students took part in the study. They first took a pre-test, then worked for 90 minutes problem solving in Peoplia, followed by a post-test. All students used the exact same system. Table 1 summarizes the obtained test scores.

Table 1. Test scores (in percent, normalized).

\begin{tabular}{|l|c|c|c|c|c|c|}
\hline & \multicolumn{2}{|c|}{ pre-test } & \multicolumn{2}{c|}{ post-test } & \multicolumn{2}{c|}{ gain } \\
\hline & mean & st.dev & mean & st.dev & mean & st.dev \\
\hline Not engaged & 0.429 & 0.245 & 0.465 & 0.283 & 0.037 & 0.283 \\
\hline Engaged & 0.439 & 0.273 & 0.562 & 0.284 & 0.123 & 0.192 \\
\hline
\end{tabular}

Students that engaged in a conversation with the tutoring agent exhibited higher learning gains. The not engaged group showed relatively low learning gain 3.7\% vs. $12.3 \%$ exhibited by the engaged group. This effect however may also be due to their previously higher motivation, and cannot be attributed to the conversation with the intelligent tutor alone. We need to further investigate the motivational state of students before the experiment, and examine the role the tutor can play, if any, in motivating students that were not motivated before.

In summary, students that engaged in social off-task dialogues with the tutor were more effective in solving problems correctly, and liked the system more (Table 2), suggesting that learning environments may produce higher learning gains by "being friends" with the students, providing them with socially relevant motivation.

Table 2. Students' answers statistics and questionnaire results.

\begin{tabular}{|l|c|c|c|c|}
\hline & Not engaged & \multicolumn{2}{c|}{ Engaged } \\
\hline & mean & st.dev & mean & st.dev \\
\hline Number of tasks attempted & 7.71 & 3.86 & 7.00 & 1.80 \\
\hline Number of tasks solved correctly & 2.85 & 1.46 & 4.00 & 2.45 \\
\hline Questions (response scale: 1=worst, 5=best) & 2.29 & 1.25 & 3.33 & 0.71 \\
\hline 1. How much did you learn in Peoplia? & 2.29 & 1.11 & 3.33 & 1.12 \\
\hline 2. How much did Peoplia help you on the post-test? & 3.14 & 1.07 & 4.33 & 1.12 \\
\hline 3. How much would you like to use Peoplia again? & 2.86 & 1.07 & 4.22 & 0.97 \\
\hline 4. How did you like Peoplia? &
\end{tabular}

Additional research needs to be conducted to evaluate the motivational benefits of introducing a socially intelligent tutoring agent. Moreover, to provide students with more in-depth social dialogues, we currently explore the tractability of extending the conversational capabilities considerably by bootstrapping its strategy from Wizard-of$\mathrm{Oz}$ data produced by human wizards during regular use by students. 
Acknowledgments. This work was supported by the Scientific Grant Agency of SR, grant No. VG1/0508/09, the Cultural and Educational Grant Agency of SR, grant No. 028-025STU-4/2010, and it is a partial result of the Research \& Development Operational Program for the project Support of Center of Excellence for Smart Technologies, Systems and Services II, ITMS 25240120029, co-funded by ERDF.

\section{References}

1. Boyer, K.E., Phillips, R., Wallis, M., Vouk, M., Lester, J.C.: Balancing the cognitive and motivational scaffolding in tutorial dialogue. In: Proc. of the $9^{\text {th }}$ Int. Conf. on Intelligent Tutoring Systems, Montreal, Springer (2008)

2. Craig, S.D., Graesser, A.C., Sullins,J., Gholson,B.: Affect and learning: An exploratory look into the role of affect in learning. Journal of Educational Media, 29, pp.241-250 (2004)

3. Rowe, J.P., McQuiggan, S.W., Mott, B.W., Lester, J.C.: Motivation in narrative-centred learning environments. In: Proceedings of the Workshop on Narrative Learning Environments, AIED 2009, Marina del Rey, pp. 40-49 (2007)

4. McQuiggan, S.W., Rowe, J.P., Lee, S., Lester, J.C.: Story-based learning: The impact of narrative on learning experiences and outcomes. In: ITS 2008, Lecture Notes in Computer Science 5091, Springer, pp. 530-539 (2008)

5. Šimko, M., Barla, M., Bieliková, M.: ALEF: A Framework for Adaptive Web-based Learning 2.0. In IFIP Advances in Information and Communication Technology Series, World Computing Congress 2010, Springer Verlag (2010)

6. Barla, M., Bielikova, M., Bou Ezzeddinne et. al.: On the impact of adaptive test question selection for learning efficiency, Computers \& Education, Vol. 55, No. 2, 846-857 (2010)

7. Blanchard, E.G., Volfson, B., Hong, Y.J., Lajoie, S.P.: Affective Artificial Intelligent in Education: From Detection to Adaptation. In: AIED2009, IOS Press, UK (2009)

8. Tvarožek, J., Bieliková, M.: Feasibility of a Socially Intelligent Tutor. In: Intelligent Tutoring Systems 2010, Part II, LNCS 6095, Springer, pp.423-425 (2010)

9. Hartup, W.: Cooperation, close relationships, and cognitive development. In: The company they keep: Friendship in childhood and adolescence. Cambridge University Press, pp. 213237 (1996)

10.Lepper, M., Henderlong, J.: Turning "Play" into "Work" and "Work" into "Play": 25 years of Research on Intrinsic versus Extrinsic Motivation. In: Intrinsic and extrinsic motivation: The search for optimal motivation and performance, pp. 257-307 (2000)

11.Johnson, W.L.: Interaction tactics for socially intelligent pedagogical agents. In: IUI 2003, ACM Press, pp. 251-253 (2003)

12.Mayer, R.E., Johnson, W.L., Shaw, E., Sandhu, S.: Constructing computer-based tutors that are socially sensitive: Politeness in educational software. Int. Journal of Human-Computer Studies. 64(1), pp.36-42 (2006)

13.McLaren, B.,M., Lim, S., Yaron, D., Koedinger, K.R,: Can a Polite Intelligent Tutoring System Lead to Improved Learning Outside of the Lab? In: AIED2007, pp.331-338 (2007)

14.Wang, N., Johnson, W.L., Mayer et al: The politeness effect: Pedagogical agents and learning outcomes. Int. Journal of Human-Computer Studies, 66, pp.98-112 (2008)

15.Malone, E., Crumlish, Ch.: Designing Social Interfaces: Principles, Best Practices and Patterns for Designing the Social Web, O'Reilly Media (2009)

16.Tvarožek, J., Kravčík, M., Bieliková, M.: Towards Computerized Adaptive Assessment Based on Structured Tasks. In: Adaptive Hypermedia 2008, LNCS 5149, Springer, pp. 224234 (2008) 